\title{
The Correlation between Hypertensive Intracerebral Hemorrhage and Internal Carotid Atherosclerosis Investigated by Carniocervial CTA
}

\author{
Bo Wu' ${ }^{1}$ Chizhong Zhou ${ }^{2 *}$ \\ ${ }^{1}$ Clinical Medicine College of Wuhan University of Science and Technology, Wuhan, China \\ ${ }^{2}$ Department of Neurosurgery, Affiliated Hospital of Wuhan University of Science and Technology, Wuhan, China \\ Email:*13407194673@139.com
}

How to cite this paper: $\mathrm{Wu}, \mathrm{B}$. and Zhou, C.Z. (2020) The Correlation between Hypertensive Intracerebral Hemorrhage and Internal Carotid Atherosclerosis Investigated by Carniocervial CTA. International Journal of Clinical Medicine, 11, 228-235. https://doi.org/10.4236/ijcm.2020.115023

Received: April 15, 2020

Accepted: April 27, 2020

Published: April 30, 2020

Copyright ( 2020 by author(s) and Scientific Research Publishing Inc. This work is licensed under the Creative Commons Attribution International License (CC BY 4.0).

http://creativecommons.org/licenses/by/4.0/

(c) (i) Open Access

\begin{abstract}
Objective: To investigate the relationship between hypertensive intracerebral hemorrhage and internal carotid atherosclerosis and its risk factors by CTA (Computed tomography angiography). Methods: The clinical materials of hypertensive intracerebral hemorrhage patients with carniocervial CTA from January 2018 to August 2019 in Puren Hospital of Wuhan were analyzed retrospectively. The correlation and risk factors between hypertensive intracerebral hemorrhage and internal carotid atherosclerosis were studied by logistic regression and descriptive analysis, at the same time, the application value of carniocervial CTA in patients with cerebral hemorrhage was evaluated. Results: There was a correlation between hypertensive intracerebral hemorrhage and internal carotid atherosclerosis $\left(\chi^{2}=5.319, P=0.021<0.05\right.$, $\mathrm{OR}=2.70>1$ ), which indicated that internal carotid atherosclerosis was the risk factor of hypertensive intracerebral hemorrhage, and there was no significant correlation between the location of internal carotid atherosclerosis, multiple atherosclerosis of internal carotid artery and hypertensive intracerebral hemorrhage. Monofactor analysis showed that the risk factors of hypertensive intracerebral hemorrhage with internal carotid atherosclerosis were sex, age, diabetes and hyperlipidemia. According to the logistic regression analysis, hyperlipidemia and diabetes were independent risk factors for hypertensive. Conclusion: The occurrence of hypertensive intracerebral hemorrhage is related to internal carotid atherosclerosis and is affected by many factors. Carniocervial CTA is helpful to the diagnosis of cerebral hemorrhage.
\end{abstract}




\section{Keywords}

Hypertensive Intracerebral Hemorrhage, Internal Carotid Atherosclerosis, Computed Tomography Angiography

\section{Background}

Hypertensive intracerebral hemorrhage is a common cerebrovascular disease in clinical work. As the problem of aging population becomes more and more prominent, the incidence of the disease is also increasing year by year. It has the characteristics of disability rate, mortality rate, high recurrence rate and is one of the biggest threats to human health in the 21st century [1]. Studies have shown that carotid atherosclerosis exists in $20 \%$ of patients with intracerebral hemorrhage [2]. This paper uses head and neck CTA, to further explore the relationship between the characteristics of carotid atherosclerotic plaque in internal carotid artery and hypertensive intracerebral hemorrhage, and expounds the application value of CTA in intracerebral hemorrhage disease.

\section{Objects and Methods}

\subsection{Object of Study}

From January 2018 to August 2019, 50 patients with hypertensive intracerebral hemorrhage who were admitted to Puren Hospital of Wuhan City were selected as the experimental group and 50 patients with non-cranial hemorrhage were selected as the control group, including 9 patients with mild craniocerebral trauma, 20 patients with dizziness and headache, 21 patients with transient ischemic attack. All patients underwent CTA examination.

Inclusion criteria: 1) consistent with the clinical diagnostic guidelines related to hypertensive intracerebral hemorrhage [3]; 2) confirmed by MRI, CT and other imaging examinations; 3 ) initial onset.

Exclusion criteria: 1) patients with secondary intracerebral hemorrhage, such as aneurysm rupture, cerebrovascular malformation, trauma, tumor, etc.; 2) patients with recurrent hypertensive intracerebral hemorrhage; 3) patients with hemorrhagic cerebral infarction; 4) patients with head and neck CTA with poor imaging and incomplete clinical data.

From January 2018 to August 2019, 50 patients with non-cranial hemorrhage were selected as the control group, including 9 patients with mild craniocerebraltrauma, 20 patients with dizziness and headache, 21 patients with transient ischemic attack, and head and neck CTA were performed during hospitalization or during outpatient visits.

\subsection{Methods}

\subsubsection{Instruments and Reagents}

Adopt 128 row GE optima660CT machine, non-ionic contrast agent iodohydrol; 
the patient has signed the consent before the examination.

\subsubsection{Scanning Mode}

Take the supine position, use the head frame to fix the head, ask the subject to keep the head and neck still during the examination, fix the forehead with bandages, ask the patient to breathe calmly, avoid swallowing when scanning. scanning range from the aortic arch to the cranial top. scan parameters: tube voltage $100 \mathrm{KV}$, tube current $350 \mathrm{~mA}$, scan layer thickness, layer interval scan $0.6 \mathrm{~mm}$ scan field of view $400, \mathrm{LW}=40$, pitch 0.984:1.000. Iodine sea-alcohol, iodine contrast agent g/50ml. 17.5 all patients were treated with low-dose group injection: through the elbow intravenous injection of contrast agent $15 \mathrm{ml}$ and saline $20 \mathrm{ml}$ flush tube, injection speed $4-5 \mathrm{ml} / \mathrm{s}$, delay Xs (x represents the scan delay time, the routine set to $10 \mathrm{~s}$ ) after the $\mathrm{C} 4$ vertebral lam dynamic scan. The micro-calcification region of interest (MROI) analysis software was used to observe and measure the peak concentration of the contrast agent at the $\mathrm{C} 4$ carotid artery, and obtain the turning point $\mathrm{Y}$ on the time-density curve of contrast enhancement Value, according to the conventional method $(\mathrm{X}+2 \mathrm{Y}+1)$ to calculate the enhanced scan delay time, wait for $5 \mathrm{~min}$ after injecting $50 \mathrm{ml}$ of contrast agent and $50 \mathrm{ml}$ of saline to perform a two-phase scan of the same site with the contrast agent injection flow rate, and obtain a head and neck CTA image.

\section{Basic Information}

Record the patient's age, gender, diabetes, hyperlipidemia, smoking, drinking history and other data. Carotid atherosclerotic plaque is defined as local bulge thickening, protruding into the lumen, thickness $\geq 1.3 \mathrm{~mm}$ [4]. According to carniocervial CTA imaging data and the 7-segment method of internal carotid artery proposed by Bouthillieretal in 1996 [5], the carotid atherosclerosis was divided into extracranial atherosclerosis and intracranial atherosclerosis.

\section{Statistical Processing}

SPSS17.0 was used for statistical analysis. Counting data is expressed in (n\%), using $\chi^{2}$ test. The measurement data is expressed by $(X \pm S)$, and $t$ test is used. Multivariate correlation analysis was performed using binary classification Logistic regression analysis. $\mathrm{P}<0.05$ is a significant difference, with statistical significance.

\section{Result}

\subsection{Comparison of Basic Clinical Data}

The patients in the hypertensive cerebral hemorrhage group were $36-81$ years old, with an average of $(62.86 \pm 10.38)$ years old. There were 32 male patients and 18 female patients. The patients in the non-cerebral hemorrhage group were 27 - 82 years old, with an average of $(59.86 \pm 13.23)$ years old. There were 26 male patients and 24 female patients. Two independent sample $t$ tests were car- 
ried out on the ages of the two groups. The $t$ value was 1.261 and the $p$ value was 0.210 . There was no difference in age between the two groups. Chi-square test was conducted on the gender composition of the two groups, and their $\chi^{2}$ value was 1.478 and $\mathrm{p}$ value was 0.224 . There was no difference in gender composition between the two groups. There was no statistically significant difference in age and gender between the two groups, and they were comparable.

\subsection{Analysis of the Incidence of Internal Carotid Atherosclerosis in Patients with Hypertensive Cerebral Hemorrhage and the Correlation between Internal Carotid Atherosclerosis and Hypertensive Cerebral Hemorrhage}

According to the imaging data of the patient's head and neck CTA, the statistics of internal carotid atherosclerotic plaque sclerosis. Among the 50 patients with hypertensive cerebral hemorrhage, 38 patients $(76.0 \%)$ with internal carotid atherosclerosis, including 11 patients $(28.9 \%)$ with internal carotid artery intracranial segment alone There were 6 patients $(15.8 \%)$ with plaque in the extracranial segment of arteries, and 21 patients (55.3\%) had both; in the control group, there were 27 patients (54\%) with internal carotid atherosclerosis. Among them, 13 patients (48.1\%) had plaque in the intracranial segment of the internal carotid artery alone, and 3 patients $(11.1 \%)$ had plaque in the extracranial segment of the internal carotid artery (11.40\%). The formation of internal carotid atherosclerosis is a risk factor for hypertensive cerebral hemorrhage $(\mathrm{P}<0.05, \mathrm{OR}==2.70>1)$. There is no difference in the probability of plaques in the intracranial and extracranial segments of the internal carotid artery leading to cerebral hemorrhage $(P>0.05)$. Not relevant $(p>0.05)$. See Tables $1-3$ for details.

\subsection{Single Factor Analysis of Carotid Atherosclerosis in Patients with Hypertensive Cerebral Hemorrhage}

Among 50 cases of hypertensive cerebral hemorrhage, patients with internal

Table 1. Correlation analysis of internal carotid atherosclerosis and hypertensive cerebral hemorrhage.

\begin{tabular}{|c|c|c|c|c|c|}
\hline & & $\begin{array}{l}\text { With internal Carotid } \\
\text { atherosclerosis }\end{array}$ & $\begin{array}{c}\text { Without internal } \\
\text { Carotid atherosclerosis }\end{array}$ & $x^{2}$ & $\mathbf{P}$ \\
\hline \multirow{3}{*}{$\begin{array}{l}\text { Hypertensive cerebral } \\
\text { hemorrhage }\end{array}$} & With & 38 & 12 & & \\
\hline & & & & 5.319 & 0.021 \\
\hline & Without & 27 & 23 & & \\
\hline
\end{tabular}

Table 2. Correlation between different parts of internal carotid artery and hypertensive cerebral hemorrhage.

\begin{tabular}{lccccc}
\hline & & Intracranial segment & Extracranial segment & $\chi^{2}$ & P \\
\hline $\begin{array}{c}\text { Hypertensive cerebral } \\
\text { hemorrhage }\end{array}$ & With & 32 & 27 & 0.340 & 0.560 \\
& Without & 24 & & & \\
\hline
\end{tabular}


Table 3. Correlation between the features of internal carotid atherosclerosis site and hypertensive cerebral hemorrhage.

\begin{tabular}{|c|c|c|c|c|c|}
\hline & & Simple part & Mixed parts & $x^{2}$ & $\mathbf{P}$ \\
\hline & With & 17 & 21 & & \\
\hline \multirow[t]{2}{*}{$\begin{array}{l}\text { Hypertensive cerebral } \\
\text { hemorrhage }\end{array}$} & & & & 1.332 & 0.248 \\
\hline & Without & 16 & 11 & & \\
\hline
\end{tabular}

carotid atherosclerosis were divided into group $\mathrm{A}$, and patients without internal carotid atherosclerosis were divided into group B. There are 38 people in group A and 12 people in group B. Univariate analysis showed that the risk factors for atherosclerosis in patients with hypertensive cerebral hemorrhage were gender, age, diabetes, smoking, hyperlipidemia and so on. The specific situation is shown in Table 4.

\subsection{Multivariate Logistic Regression Analysis of Hypertensive Cerebral Hemorrhage}

With or without hypertensive intracerebral hemorrhage (with hypertensive intracerebral hemorrhage $=1$, without hypertensive intracerebral hemorrhage $=0$ ) as the dependent variable, gender (male $=1$, female $=0)$, age ( $\geq 60$ is $1,<60$ is 0$)$, diabetes (yes $=1$, no $=0)$, smoking $(\mathrm{y}=1$, no $=0)$, hyperlipidemia $(\mathrm{y}=1$, no $=$ 0 ) and other factors are independent variables, and multivariate logistic is performed after assignment regression analysis. Hyperlipidemia and diabetes are independent risk factors for hypertensive cerebral hemorrhage. The specific situation is shown in Table 5.

\section{Discussion}

Atherosclerosis is a common cause of cerebrovascular disease. $70 \%$ to $80 \%$ of blood in the brain is supplied by the internal carotid artery. Previous studies have pointed out that carotid atherosclerotic plaque is a high factor for cerebral infarction. However, regarding carotid artery, there are few studies on the correlation between atherosclerosis and cerebral hemorrhage. This article uses the head and neck CTA to explain the correlation and risk factors of internal carotid atherosclerotic plaque with hypertensive cerebral hemorrhage.

This study found that the formation of internal carotid atherosclerotic plaques is a risk factor for hypertensive intracerebral hemorrhage, but it has no significant correlation with whether the plaques are in the intracranial or extracranial segment of the internal carotid artery. Hypertensive cerebral hemorrhage is based on hypertension, and various types of hypertension can cause cerebral hemorrhage under certain circumstances. At the same time, studies have shown that the incidence of atherosclerosis in patients with hypertension is significantly increased. Patients with hypertension have atherosclerosis $3-4$ times higher than those with normal blood pressure. May be due to high blood pressure, the arterial wall is under high pressure, increasing the excitability of its sympathetic 
Table 4. Single factor analysis of carotid atherosclerosis in patients with hypertensive cerebral hemorrhage.

\begin{tabular}{|c|c|c|c|c|c|}
\hline relevant factor & classification & Group A & Group B & $x^{2}$ & $\mathbf{P}$ \\
\hline \multirow{3}{*}{ Gender } & Male & 28 & 4 & \multirow{3}{*}{4.813} & \multirow{3}{*}{0.028} \\
\hline & & & & & \\
\hline & Female & 10 & 8 & & \\
\hline \multirow[b]{2}{*}{ Age } & $\geq 60$ & 28 & 3 & \multirow[b]{2}{*}{7.225} & \multirow[b]{2}{*}{0.007} \\
\hline & $<60$ & 10 & 9 & & \\
\hline \multirow{3}{*}{ Diabetes } & With & 22 & 2 & \multirow{3}{*}{6.211} & \multirow{3}{*}{0.013} \\
\hline & & & & & \\
\hline & Without & 16 & 10 & & \\
\hline \multirow[b]{2}{*}{ Hyperlipidemia } & With & 30 & 5 & \multirow[b]{2}{*}{4.31} & \multirow[b]{2}{*}{0.036} \\
\hline & Without & 15 & 7 & & \\
\hline \multirow{3}{*}{ Smoking } & With & 27 & 3 & \multirow{4}{*}{6.255} & \multirow{3}{*}{0.012} \\
\hline & & & & & \\
\hline & Without & 11 & 9 & & \\
\hline \multirow[b]{2}{*}{ Drinking } & With & 19 & 8 & & \\
\hline & Writhout & 10 & 4 & 1.020 & 0.313 \\
\hline
\end{tabular}

Note: Group A: patients with internal carotid atherosclerosis among 50 cases of hypertensive cerebral hemorrhage; Group B: patients without internal carotid atherosclerosis among 50 cases of hypertensive cerebral hemorrhage.

Table 5. Multivariate logistic regression analysis of hypertensive cerebral hemorrhage.

\begin{tabular}{cccccccc}
\hline & B & S.E, & Wals & df & Sig. & Exp (B) & EXP(B) 95\% C.I. \\
\hline Gender & 0.053 & 0.469 & 0.013 & 1 & 0.909 & 1.055 & $0.042-2.647$ \\
Age & -0.284 & 0.480 & 0.352 & 1 & 0.553 & 0.752 & $0.294-1.926$ \\
Hyperlipidemia & 0.916 & 0.449 & 4.163 & 1 & 0.041 & 2.500 & $1.037-6.027$ \\
Diabetes & 1.221 & 0.499 & 5.996 & 1 & 0.014 & 3.391 & $1.276-9.010$ \\
Smoking & 0.526 & 0.445 & 1.397 & 1 & 0.237 & 1.692 & $0.707-4.050$ \\
constant & -1.075 & 0.489 & 4.841 & 1 & 0.028 & 0.341 & \\
\hline
\end{tabular}

nerves, increasing the amount of adrenaline angiotensin, endothelial cell damage, causing excessive prostaglandins and thromboxane to adhere to the patient's platelets And lipid deposition, and stimulate smooth muscle cell proliferation, eventually leading to the formation of carotid atherosclerosis [6]. In this study, it was found that in the hypertensive cerebral hemorrhage group and the control group, the incidence of atherosclerosis in the intracranial segment of the internal carotid artery was higher than that in the extracranial segment, respectively, $64 \%$ and $48 \%$. The specific reason is not clear, and it may be caused by the relatively tortuous blood vessels of the internal carotid artery from the rock section to the communication section in the cranial, resulting in relatively unstable hemodynamics.

Multivariate analysis of whether patients with hypertensive cerebral hemorrhage have internal carotid atherosclerosis found that age greater than 60 years 
old, hyperlipidemia, diabetes, smoking, and gender are all influencing factors. And multivariate Logistic regression analysis of hypertensive cerebral hemorrhage pointed out that hyperlipidemia $(\mathrm{OR}=2.500,95 \% \mathrm{CI}: 1.037-6.027, \mathrm{P}=$ $0.041)$ and diabetes $(\mathrm{OR}=3.391,95 \% \mathrm{CI}: 1.276-9.010, \mathrm{P}=0.014)$ is an independent risk factor for hypertensive cerebral hemorrhage. Therefore, for hypertensive patients over 60 years old, in addition to actively controlling blood pressure, blood lipid and blood sugar levels should also be paid attention to. It has positive significance for preventing hypertensive cerebral hemorrhage.

With the development of CT angiography (Computed tomography angiogram, CTA) technology, we can use CTA image data to observe and measure the plaque in the blood vessels of the head and neck and related data. Some research results show that in detecting the number of internal carotid artery plaques, the results of ultrasound examination are significantly lower than the results of CTA examinations. The relationship between the characteristics of plaque and hypertensive cerebral hemorrhage. CT angiography of the head and neck is one of the important examination methods of cerebrovascular disease. Its operation is simple, the result is faster, safe and efficient, and it can accurately display the position, size, and shape of the examiner's blood vessel and surrounding tissue. The doctor provides a reliable basis for the diagnosis of the disease [7]. For patients with cerebral hemorrhage, CTA examination can further investigate cerebral hemorrhage caused by aneurysm and arteriovenous malformation. Compared with ultrasound, the imaging of blood vessels by CTA is clearer, especially for the examination of intracranial blood vessels.

\section{Conclusion}

In summary, patients with high blood pressure and diabetes who are over 60 years old are actively controlling blood lipid levels and quitting smoking, which is one of the important measures to prevent hypertensive cerebral hemorrhage. Reducing the prevalence of carotid atherosclerosis has certain significance for controlling hypertensive cerebral hemorrhage. As an auxiliary examination method of cerebrovascular disease, CTA is conducive to improving the accuracy of diagnosis and is worthy of promotion in the clinic.

\section{Conflicts of Interest}

The authors declare no conflicts of interest regarding the publication of this paper.

\section{References}

[1] Chamarro, R., Ponz, A., Alonso, M.D., Gil, R. and Laínez, J.M. (2003) Arterial Hypertension and Intracerebral Hemorrhage. Neurologia, 18.

[2] Shoichiro, S., Toshiyuki, U., Mikito, H., Kazuyuki, N., Kazuo, M. and Kazunori, T. (2013) Intra- and Extracranial Atherosclerotic Disease in Acute Spontaneous Intracerebral Hemorrhage. Journal of the Neurological Sciences, 332, 116-120. https://doi.org/10.1016/j.jns.2013.06.031 
[3] Broderick, J., Connolly, S., Feldmann, E., et al. (2007) Guidelines for the Management of Spontaneous Intracerebral Hemorrhage in Adults. 2007 Update: A Guideline from the American Heart Association/American Stroke Association Stroke Council, High Blood Pressure Research Council, and the Quality of Care and Outcomes in Research Interdisciplinary Working Group. The American Academy of Neurology Affirms the Value of This Guideline as an Educational Tool for Neurologists. Chinese Journal of Cerebrovascular Diseases (Electronic Version), 15, 484-504. https://doi.org/10.1161/STROKEAHA.107.183689

[4] Yuan, Y.M., Sun, M.M. and Hang, X.Y. (2015) Relationship between the Ambulatory Blood Pressure Index and Atheroscle-Rosis in Elderly Patients with Hypertension. China Medical Herald, 20, 117-120.

[5] Bouthillier, A., van Loveren, H.R. and Keller, J.T. (1996) Segments of the Internal Carotid Artery: A New Classification. Neurosurgery, 38, 425-433.

https://doi.org/10.1227/00006123-199603000-00001

[6] Liu, Z.M., Chen, Q.X., Tian, D.F., Wang, L., Liu, B.H. and Zhang, S.Q. (2015) Clinical Significance of Dynamic Monitoring by Transcranial Doppler Ultrasound and Intracranial Pressure Monitor after Surgery of Hypertensive Intracerebral Hemorrhage. International Journal of Clinical and Experimental Medicine, 8.

[7] Walter, C.M., Dumont, T.M., Zaninovich, O.A., et al. (2017) Completion of the Circle of Willis Varies by Gender, Age, and Indication for Computed Tomography Angiography. World Neurosurgery, 106, 953-963.

https://doi.org/10.1016/j.wneu.2017.07.084 